\title{
LIMIT THEOREMS FOR A MINIMAL RANDOM WALK MODEL
}

\author{
CRISTIAN F. COLETTI, RENATO J. GAVA AND LUCAS R. DE LIMA
}

\begin{abstract}
We study the minimal random walk introduced by Kumar, Harbola and Lindenberg 12 . It is a random process on $\{0,1, \ldots\}$ with unbounded memory which exhibits subdiffusive, diffusive and superdiffusive regimes. We prove the law of large numbers for the whole parameter set. Then we prove the central limit theorem and the law of the iterated logarithm for the minimal random walk under diffusive and marginally superdiffusive behaviors. More interestingly, we establish a result for the minimal random walk when it possesses the three regimes; we show the convergence of its rescaled version to a non-normal random variable.
\end{abstract}

\section{INTRODUCTION}

In this work we are concerned with the minimal random walk model introduced in [12, a random walk $X_{n}$ on $\{0,1,2, \ldots\}$ with dependent increments and unbounded memory such that at each step the walker either moves to the nearest neighbour to its right or remains at the same place. The goal of Kumar, Harbola and Lindenberg [12 was to introduce a model, as simple as possible, in which the exact computation of the first two moments, depending on the choice of a pair of parameters $0 \leq q \leq 1$ and $0 \leq p \leq 1$, enables us to view the emergence of subdiffusive, diffusive and superdiffusive regimes.

Some years before the appearance of the minimal model proposed in [12], the same authors introduced the lazy elephant random walk [11, a generalization of the so-called elephant random walk proposed by Schütz and Trimper [15]. This generalized model, like the one treated in this article, also exhibits the three different diffusion regimes; it allows the walker to go forward, backward or remain at rest. Therefore the random walk we deal with in this work is simpler: the increments have only two possible choices, either a forward step or a resting step. All these models are non-Markovian since their past or memory matters in the future steps of the walk. In the last two decades, random walks with unbounded memory has received considerable attention from the physics community as one can see in [4, 7, 9, 11,15$]$ and references therein. More recently some mathematical papers $[2,3,5,6$ have established

Date: August 27, 2019.

R.J.G. thanks FAPESP (grants 2017/10555-0 and 2018/04764-9).

C.F.C. thanks FAPESP grant 2017/10555-0.

L. R. L. thanks CAPES - Finance Code 001. 
rigorously the first limit theorems on the subject. Regarding interacting non-Markovian random walks it is worth mentioning that the authors of [1, 10 considered the influence of memory in such systems. In both works the authors study how memory affects the behavior of an interacting particle system and its relation to non-Markovian exclusion processes.

Here we establish a series of results for the minimal random walk 12 . First, we prove a strong law of large numbers (Theorem 1), then we prove a central limit theorem (Theorem 2) and the law of the iterated logarithm (Theorem 3 ) for an appropriate choice of parameters at which the model shows diffusive and marginally superdiffusive behaviors. Finally, in Theorem 4. we state our main result. We address the case in which $q=0$ where the random walk $X_{n}$ exhibits subdiffusion, diffusion and superdiffusion regimes depending on whether $p<1 / 2$, $p=1 / 2$ or $p>1 / 2$ respectively. We show that the rescaled random walk $X_{n}$ converges to a non-degenerate non-normal random variable for any $p \in(1 / 2,1)$. In order to show that it does not obey a normal distribution we compute the first four moments of the limit random variable. It remains open to find out the correct scaling of $X_{n}$ in order to obtain convergence for $q=0$ and $p \leq 1 / 2$. The proofs given here are based on a martingale approach 8, 16, 17]. The martingale theory is an extension of the theory of stochastic processes with independent increments, allowing dependency among them. Given a sequence $\left\{M_{n}\right\}_{n \geq 0}$ and all the information $\mathcal{F}_{n}$ on this sequence up to time $n$, we say that $M_{n}$ is a martingale if

$$
\mathbb{E}\left(M_{n+1} \mid \mathcal{F}_{n}\right)=M_{n}
$$

In other words, conditioned on the history up to time $n$, the average value at $M_{n+1}$ is equal to $M_{n}$. A nice introduction to the subject can be found in Williams [18].

The paper is organized as follows. In the next section we define the model formally. In Section 3 we state the main results of this work. We begin Section 4 with some preliminaries results and then we present the proofs of the main theorems.

\section{Definition OF THE MODEL}

We now define the minimal random walk as follows. It starts at $X_{0}=0$. At each discrete time step the walker moves one step to the right or stays put. Therefore

$$
X_{n+1}=X_{n}+\eta_{n+1}
$$

where $\eta_{n+1} \in\{0,1\}$ is a random variable. The memory consists of the set of random variables $\eta_{n^{\prime}}$ at previous time steps which the walker remembers as follows.

Initially, the walker jumps to the right with probability $s$ and remains at 0 with probability $1-s$. At time $n+1$, for $n \geq 1$, a number $n^{\prime}$ is chosen from the set $\{1,2, \ldots, n\}$ uniformly at 
random. Then $\eta_{n+1}$ is determined stochastically by the following rule. If $\eta_{n^{\prime}}=1$, then

$$
\eta_{n+1}=\left\{\begin{array}{ll}
1 & \text { with probability } p \\
0 & \text { with probability } 1-p
\end{array} .\right.
$$

If $\eta_{n^{\prime}}=0$, then

$$
\eta_{n+1}=\left\{\begin{array}{ll}
1 & \text { with probability } q \\
0 & \text { with probability } 1-q
\end{array} .\right.
$$

It turns out from the definition that $X_{n}=\sum_{k=1}^{n} \eta_{k}$ and that

$$
\begin{aligned}
\mathbb{P}\left[\eta_{n+1}=\eta \mid \eta_{1}, \ldots, \eta_{n}\right] & =\frac{1}{n} \sum_{k=1}^{n}\left[1-\eta+(2 \eta-1)\left(q+\alpha \eta_{k}\right)\right] \\
& =1-\eta+(2 \eta-1)\left(q+\alpha \frac{X_{n}}{n}\right) \quad \text { for } n \geq 1,
\end{aligned}
$$

where $\eta \in\{0,1\}$ and $\alpha=p-q$.

The expectation of the increment $\eta_{n+1}$ is given by

$$
\mathbb{E}\left[\eta_{n+1}\right]=q+\alpha \frac{\mathbb{E}\left[X_{n}\right]}{n} \text { for } n \geq 1 .
$$

In $[12]$ the authors showed that

$$
\begin{aligned}
\mathbb{E}\left[X_{n}\right] & =\frac{q n}{1-\alpha}+\left(s-\frac{q}{1-\alpha}\right) \frac{\Gamma(n+\alpha)}{\Gamma(1+\alpha) \Gamma(n)} \\
& \sim \frac{q n}{1-\alpha}+\left(s-\frac{q}{1-\alpha}\right) \frac{n^{\alpha}}{\Gamma(1+\alpha)} \quad \text { for } \quad n \gg 1
\end{aligned}
$$

\section{MAIN RESUlts}

Theorem 1. Let $\left(X_{n}\right)_{n \geq 1}$ be the minimal random walk described above. Then

$$
\lim _{n \rightarrow \infty} \frac{X_{n}-\mathbb{E}\left[X_{n}\right]}{n}=0 \quad \text { a.s. }
$$

for any value of $\alpha \in[-1,1)$. In other words,

$$
\lim _{n \rightarrow \infty} \frac{X_{n}}{n}=\frac{q}{1-\alpha} \quad \text { a.s. }
$$

Remark 1. The case $\alpha=1$ is not covered by the strong law of large numbers(SLLN). Indeed, when $p=1$ and $q=0$, the walk is trivial since by definition its dynamics is determined by the first step $\eta_{1}$, that is, $\eta_{n}=\eta_{1}$ for all $n \geq 1$. Hence $X_{n} / n$ reduces to a binary random variable.

Theorem 2. Let $\left(X_{n}\right)_{n \geq 1}$ be the minimal random walk and let $\alpha \leq 1 / 2, p<1$ and $q>0$. 
a) If $\alpha<1 / 2$, then

$$
\frac{X_{n}-\frac{q}{1-\alpha} n}{\sqrt{n}} \stackrel{d}{\rightarrow} N\left(0, \frac{q(1-p)}{(1-\alpha)^{2}(1-2 \alpha)}\right) .
$$

b) If $\alpha=1 / 2$, then

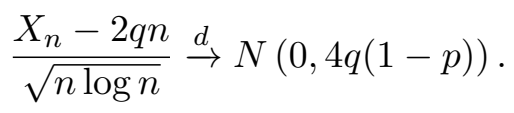

Theorem 3. Let $\left(X_{n}\right)_{n \geq 1}$ be the minimal random walk and let $\alpha \leq 1 / 2, p<1$ and $q>0$.

a) If $\alpha<1 / 2$, then

$$
\limsup _{n \rightarrow \infty} \frac{\left|X_{n}-\frac{q n}{1-\alpha}\right|}{\sqrt{2 n \log \log n}}=\sqrt{\frac{q(1-p)}{(1-\alpha)^{2}(1-2 \alpha)}} \text { a.s. }
$$

b) If $\alpha=1 / 2$, then

$$
\limsup _{n \rightarrow \infty} \frac{\left|X_{n}-2 q n\right|}{\sqrt{2 n \log n \log \log \log n}}=\sqrt{4 q(1-p)} \text { a.s. }
$$

We now state our main result. It deals with the case $q=0$ and $1 / 2<p<1$ where the random walk exhibits a superdiffusion regime.

Theorem 4. Let $\left(X_{n}\right)_{n \geq 1}$ be the minimal random walk. If $q=0$ and $1 / 2<p<1$, then

$$
\frac{X_{n}}{n^{p} \Gamma(1+p)^{-1}}-s \rightarrow M \text { a.s. and in } L^{m} \text { for } m \geq 1,
$$

where $M$ is a non-normal random variable such that

$$
\begin{aligned}
\mathbb{E}(M) & =0 \\
\mathbb{E}\left(M^{2}\right) & =\frac{2 s \Gamma(1+p)^{2}}{\Gamma(1+2 p)}-s^{2} \\
\mathbb{E}\left(M^{3}\right) & =\frac{6 s \Gamma(1+p)^{3}}{\Gamma(1+3 p)}-\frac{6 s^{2} \Gamma(1+p)^{2}}{\Gamma(1+2 p)}+2 s^{3} \\
\mathbb{E}\left(M^{4}\right) & =\frac{24 s \Gamma(1+p)^{4}}{\Gamma(1+4 p)}-\frac{24 s^{2} \Gamma(1+p)^{3}}{\Gamma(1+3 p)}+\frac{12 s^{3} \Gamma(1+p)^{2}}{\Gamma(1+2 p)}-3 s^{4} .
\end{aligned}
$$

Remark 2. We note that the same approach employed in the proof of Theorem 4 works when the parameters are $q>0$ and $\alpha>1 / 2$ and the model exhibits superdiffusion behavior. This occurs, basically, because the variance of the martingale $M_{n}$, which is related to $X_{n}$ and is introduced in Subsection 4.1. converges as $n \rightarrow \infty$. 


\section{Proofs}

4.1. Preliminaries. We know from (1) that

$$
\mathbb{P}\left[\eta_{n+1}=1 \mid \eta_{1}, \ldots, \eta_{n}\right]=q+\alpha \frac{X_{n}}{n} .
$$

Put

$$
a_{1}=1 \quad \text { and } \quad a_{n}=\prod_{j=1}^{n-1}\left(1+\frac{\alpha}{j}\right) \text { for } n \geq 2 \text { and } \alpha>-1 .
$$

Note that

$$
a_{n}=\frac{\Gamma(n+\alpha)}{\Gamma(n) \Gamma(\alpha+1)} \sim \frac{n^{\alpha}}{\Gamma(1+\alpha)} \text { as } n \rightarrow \infty
$$

and that $a_{n} \rightarrow \infty$ as $n \rightarrow \infty$ if $\alpha>0, a_{n}=1$ for $n \geq 1$ if $\alpha=0$, and $a_{n} \rightarrow 0$ if $\alpha<0$.

Define the filtration $\mathcal{F}_{n}=\sigma\left(\eta_{1}, \ldots, \eta_{n}\right)$ and $M_{n}=\frac{X_{n}-\mathbb{E}\left[X_{n}\right]}{a_{n}}$ for $n \geq 1$. We claim that $\left\{M_{n}\right\}_{n \geq 1}$ is a martingale with respect to $\left\{\mathcal{F}_{n}\right\}_{n \geq 1}$, for

$$
\begin{aligned}
\mathbb{E}\left[M_{n+1} \mid \mathcal{F}_{n}\right] & =\frac{\left(X_{n}-\mathbb{E}\left[X_{n}\right]\right)}{a_{n+1}}+\frac{\mathbb{E}\left[\eta_{n+1} \mid \mathcal{F}_{n}\right]-\mathbb{E}\left[\eta_{n+1}\right]}{a_{n+1}} \\
& =\frac{\left(X_{n}-\mathbb{E}\left[X_{n}\right]\right)}{a_{n+1}}+\frac{\alpha \frac{X_{n}}{n}-\alpha \frac{\mathbb{E}\left[X_{n}\right]}{n}}{a_{n+1}} \\
& =\frac{\left(X_{n}-\mathbb{E}\left[X_{n}\right]\right)}{a_{n+1}}+\frac{\frac{\alpha}{n}\left(X_{n}-\mathbb{E}\left[X_{n}\right]\right)}{a_{n+1}} \\
& =\left(X_{n}-\mathbb{E}\left[X_{n}\right]\right) \frac{\left(1+\frac{\alpha}{n}\right)}{a_{n+1}} \\
& =M_{n} .
\end{aligned}
$$

Note that (4) amounts to $\mathbb{P}\left(\eta_{n+1}=1 \mid \mathcal{F}_{n}\right)=q+\alpha \frac{X_{n}}{n}$.

The walker jumps to the right at the $n$-th step time with probability $p_{n}=\mathbb{E}\left[\eta_{n}\right]$. Then combining (1) and (3), we have that

$$
p_{n}=\frac{q}{1-\alpha}+\alpha \frac{a_{n-1}}{n-1}\left(s-\frac{q}{1-\alpha}\right) \text { for } \alpha<1
$$

A direct consequence of (5) is the following result.

Lemma 1. The series $\sum_{n=1}^{\infty} \frac{1}{a_{n}^{2}}$ converges if and only if $\alpha>1 / 2$. 
Let $\left(D_{n}\right)_{n \geq 1}$ be the martingale differences defined by $D_{1}=M_{1}$ and, for $n \geq 2, D_{n}=$ $M_{n}-M_{n-1}$. Observe that

$$
\begin{aligned}
D_{j} & =\frac{X_{j}-\mathbb{E}\left[X_{j}\right]}{a_{j}}-\frac{X_{j-1}-\mathbb{E}\left[X_{j-1}\right]}{a_{j-1}} \\
& =\frac{\eta_{j}-\mathbb{E}\left[\eta_{j}\right]}{a_{j}}-\frac{\left(X_{j-1}-\mathbb{E}\left[X_{j-1}\right]\right)}{j-1} \frac{\alpha}{a_{j}} .
\end{aligned}
$$

Furthermore, since the increments $\eta_{j}$ 's are uniformly bounded, it is trivial to see that

$$
\left|D_{j}\right| \leq \frac{2}{a_{j}} \text { a.s. }
$$

Let us state a well known result involving the gamma function and which will be used afterwards.

Lemma 2. For any non-negative real numbers $a$ and $b$ such that $b \neq a+1$ and for all $n \geq 1$, we have

$$
\sum_{k=1}^{n} \frac{\Gamma(k+a)}{\Gamma(k+b)}=\frac{\Gamma(n+a+1)}{(b-a-1) \Gamma(n+b)}\left(\frac{\Gamma(n+b) \Gamma(1+a)}{\Gamma(n+a+1) \Gamma(b)}-1\right) .
$$

4.2. Auxiliary results for martingale differences. In the course of the proofs of the limit theorems we will use some known results from martingale theory. We state the following theorems and their proofs can be found in the cited references. Let $\left(M_{n}, \mathcal{F}_{n}\right)_{n \geq 1}$ be a given martingale and let $\left(D_{n}\right)_{n \geq 1}$ be its associated sequence of martingale differences.

Theorem 5. [17, Theorem 3.3.1] Let $b_{j}$ be $\mathcal{F}_{j-1}$-measurable for each $j \geq 1$ such that $0<$ $b_{j} \nearrow \infty$ a.s. If

$$
\sum_{j=1}^{\infty} \frac{\mathbb{E}\left[\left|D_{j}\right|^{r} \mid \mathcal{F}_{j-1}\right]}{b_{j}^{r}}<\infty \quad \text { for some } 0<r \leq 2
$$

then

$$
M_{n} / b_{n} \rightarrow 0 \quad \text { a.s. }
$$

Theorem 6. [8, Corollary 3.1] Let $\left\{\sum_{k=1}^{i} D_{n k}, \mathcal{F}_{n i}, 1 \leq i \leq k_{n}, n \geq 1\right\}$ be a martingale array with $\mathcal{F}_{n i} \subset \mathcal{F}_{(n+1) i}, \sum_{k=1}^{i} D_{n k} \in L^{2}$ and $\mathbb{E}\left[\sum_{k=1}^{i} D_{n k}\right]=0$ for all $i \in\left\{1,2, \ldots, k_{n}\right\}$, $n \geq 1$. Let $\sigma^{2}$ be an almost surely finite random variable. If

a) for all $\varepsilon>0, \sum_{j=1}^{k_{n}} \mathbb{E}\left(D_{n j}^{2} \mathbb{I}\left(\left|D_{n j}\right|>\varepsilon\right) \mid \mathcal{F}_{j-1}\right) \stackrel{p}{\rightarrow} 0$ (conditional Lindeberg condition), where $\mathbb{I}(A)$ is the indicator function

b) $V_{n k_{n}}^{2}=\sum_{j=1}^{k_{n}} \mathbb{E}\left(D_{n j}^{2} \mid \mathcal{F}_{j-1}\right) \stackrel{p}{\rightarrow} \sigma^{2}$

then

$$
\sum_{k=1}^{k_{n}} D_{n k} \stackrel{d}{\rightarrow} Z
$$


where the characteristic function of $Z$ is given by $\phi_{Z}(t)=\mathbb{E}\left[\exp \left(-\frac{1}{2} \sigma^{2} t^{2}\right)\right]$. In particular, if $\sigma^{2}$ is almost surely a constant, $Z \stackrel{d}{=} N\left(0, \sigma^{2}\right)$.

Theorem 7. [16, Theorems 1-2] Consider a sequence of positive constants $\left(s_{n}\right)_{n \geq 1}$ such that $\lim _{n \rightarrow \infty} s_{n}=\infty$ and

$$
\frac{1}{s_{n}^{2}} \sum_{j=1}^{n} \mathbb{E}\left[D_{j}^{2} \mid \mathcal{F}_{j-1}\right] \stackrel{a . s}{\longrightarrow} 1 .
$$

Define $u_{n}=\sqrt{2 \log \log s_{n}^{2}}$. If there is a sequence of random variables $\left(K_{n}\right)_{n \geq 1}$ such that

$$
\left|D_{j}\right| \leq K_{j} \frac{s_{j}}{u_{j}} \quad \text { a.s. }
$$

where $\lim _{n \rightarrow \infty} K_{n}=0$ and each $K_{n}$ is $\mathcal{F}_{n-1}$-measurable, then

$$
\limsup _{n \rightarrow \infty} \frac{M_{n}}{s_{n} u_{n}}=1 \quad \text { a.s. }
$$

The last result which we shall need in what follows is the following:

Theorem 8. [8, Theorem 2.10] If $\left(M_{n}, \mathcal{F}_{n}\right)_{n \geq 1}$ is a martingale and $1<m<\infty$, then there exists constants $c_{1}$ and $c_{2}$ depending only on $m$ such that

$$
c_{1} \mathbb{E}\left(\left|\sum_{j=1}^{n} D_{j}^{2}\right|^{m / 2}\right) \leq \mathbb{E}\left(\left|M_{n}\right|^{m}\right) \leq c_{2} \mathbb{E}\left(\left|\sum_{j=1}^{n} D_{j}^{2}\right|^{m / 2}\right) .
$$

\subsection{Strong law of large numbers.}

Proof of Theorem 1. The result is a straightforward consequence of Theorem 5. Define $b_{i}=$ $i^{1-\alpha}$ and take $r \in(1,2)$. Now use inequality (8) to observe that the conditions of the theorem are fulfilled. Thus we arrive at the desired conclusion.

\subsection{Central limit theorem.}

Proof of Theorem 2. Define

$$
s_{n}^{2}:=\sum_{j=1}^{n} \frac{p_{j}\left(1-p_{j}\right)}{a_{j}^{2}} \text { for } n \geq 1
$$


Note that Theorem 1$]$ gives $\frac{X_{n}-\mathbb{E}\left[X_{n}\right]}{n}=\mathrm{o}(1)$ (a.s.). Now equations (1) and $(7)$ and the fact that $\eta_{j}^{2}=\eta_{j}$ a.s. allow us to conclude that

$$
\begin{aligned}
\mathbb{E}\left[D_{j}^{2} \mid \mathcal{F}_{j-1}\right] & =\frac{1}{a_{j}^{2}} \mathbb{E}\left[\left(\eta_{j}-\mathbb{E}\left[\eta_{j}\right]-\frac{X_{j-1}-\mathbb{E}\left[X_{j-1}\right]}{j-1} \alpha\right)^{2} \mid \mathcal{F}_{j-1}\right] \\
& =\frac{1}{a_{j}^{2}} \mathbb{E}\left[\eta_{j}^{2}-2 p_{j} \eta_{j}+p_{j}^{2} \mid \mathcal{F}_{j-1}\right]+\mathrm{o}\left(\frac{1}{a_{j}^{2}}\right) \\
& =\frac{p_{j}\left(1-p_{j}\right)}{a_{j}^{2}}+\mathrm{o}\left(\frac{1}{a_{j}^{2}}\right) \text { a.s. }
\end{aligned}
$$

Both claims in Theorem 2 amount to prove that

$$
\frac{X_{n}-\mathbb{E}\left[X_{n}\right]}{a_{n} s_{n}} \stackrel{d}{\rightarrow} N(0,1) .
$$

Recall that $\mathbb{E}\left(X_{n}\right) \sim \frac{q n}{1-\alpha}$ and note that [2] implies

$$
\mathbb{P}\left(\eta_{n}=1\right) \rightarrow \frac{q}{1-\alpha} \quad \text { as } n \rightarrow \infty .
$$

Combining Lemma 1 and (5) we get

$$
\begin{aligned}
s_{n}^{2} & \sim \frac{q(1-p)}{(1-\alpha)^{2}} \Gamma(1+\alpha)^{2} \frac{n^{1-2 \alpha}}{1-2 \alpha}, \text { if } \alpha<1 / 2, \\
s_{n}^{2} & \sim 4 q(1-p) \Gamma(3 / 2)^{2} \log n, \text { if } \alpha=1 / 2
\end{aligned}
$$

which in turn implies that

$$
\begin{aligned}
& a_{n} s_{n} \sim \sqrt{\frac{q(1-p) n}{(1-\alpha)^{2}(1-2 \alpha)}}, \text { if } \alpha<1 / 2 \\
& a_{n} s_{n} \sim 2 \sqrt{q(1-p) n \log n}, \quad \text { if } \alpha=1 / 2 .
\end{aligned}
$$

We now check 12 . Let us verify that the two conditions of Theorem 6 are fulfilled. Start with the conditional Lindeberg condition. Let $D_{n j}=\frac{D_{j}}{s_{n}}$ for $1 \leq j \leq n$. Given $\varepsilon>0$, we need to prove that

$$
\sum_{j=1}^{n} \mathbb{E}\left(D_{n j}^{2} \mathbb{I}\left(\left|D_{n j}\right|>\varepsilon\right) \mid \mathcal{F}_{j-1}\right) \rightarrow 0 \text { a.s. }
$$

If $\alpha \geq 0$, then $a_{n} \geq 1$ and $s_{n} \rightarrow \infty$. Next note that $\left\{\left|D_{n j}\right|>\varepsilon\right\} \subset\left\{\frac{2}{s_{n}}>\varepsilon\right\}$ a.s, but the last set is a.s. empty for $n$ large enough, so (17) holds. If $\alpha<0$, observe that $\lim _{n \rightarrow \infty} a_{n} s_{n}=\infty$ and $a_{j}^{-1} \leq a_{n}^{-1}$ for $j=1, \ldots, n$. Then it is easy to see that $\left\{\left|D_{n j}\right|>\varepsilon\right\} \subset\left\{\frac{2}{a_{n} s_{n}}>\varepsilon\right\}$ a.s. and again the latter set is a.s. empty for $n$ sufficiently large. 
Lemma 1 and (10) yield that $s_{n} \rightarrow \infty$ as $n \rightarrow \infty$ if and only if $\alpha \leq 1 / 2$. In view of this fact, (10), 11) and Theorem 1 we may claim that

$$
\frac{1}{s_{n}^{2}} \sum_{j=1}^{n} \mathbb{E}\left[D_{j}^{2} \mid \mathcal{F}_{j-1}\right] \stackrel{a . s}{\longrightarrow} 1 .
$$

In virtue of Theorem 6 we may conclude that

$$
\sum_{j=1}^{n} D_{n j}=\frac{X_{n}-\mathbb{E}\left[X_{n}\right]}{a_{n} s_{n}} \stackrel{d}{\rightarrow} N(0,1) .
$$

\subsection{Law of the iterated logarithm.}

Proof of Theorem 3. Both claims in Theorem 3 follow from a straightforward application of Theorem 7 to our random walk. Consider $K_{n}:=\frac{2 u_{n}}{a_{n} s_{n}}$; then we can verify that $\lim _{n \rightarrow \infty} K_{n}=0$ as a consequence of (13), (14), (15) and (16). Therefore, the proof of the result in item a) of Theorem 3 follows from (8), (13) and (18) and the result in item b) of the same theorem follows from (8), (14) and (18).

\subsection{Proof of Theorem 4, Notice that now}

$$
\mathbb{P}\left[\eta_{n+1}=1 \mid \mathcal{F}_{n}\right]=p \frac{X_{n}}{n} \quad \text { for } n \geq 1
$$

Equations (2) and (3) imply that $\mathbb{P}\left(\eta_{n}=1\right) \rightarrow 0$ as $n \rightarrow \infty$. Moreover, we get from (3) and (5) that

$$
\mathbb{E}\left(X_{n}\right)=s a_{n} \sim s \frac{n^{p}}{\Gamma(1+p)} .
$$

As part of the proof of Theorem 4 , we need to compute the second, the third and the fourth moments of $X_{n}$.

4.6.1. Computation of $\mathbb{E}\left(X_{n}^{2}\right), \mathbb{E}\left(X_{n}^{3}\right)$ and $\mathbb{E}\left(X_{n}^{4}\right)$. Let us start with $\mathbb{E}\left(X_{n}^{2}\right)$. Keep in mind that $\eta_{j}^{2}=\eta_{j}$ a.s., so from $(19)$ we obtain

$$
\mathbb{E}\left(X_{n+1}^{2} \mid \mathcal{F}_{n}\right)=X_{n}^{2}\left(\frac{n+2 p}{n}\right)+\frac{p X_{n}}{n},
$$

which yields

$$
\mathbb{E}\left(X_{n+1}^{2}\right)=\mathbb{E}\left(X_{n}^{2}\right)\left(\frac{n+2 p}{n}\right)+p \frac{\mathbb{E}\left(X_{n}\right)}{n} .
$$

Next we obtain by induction that

$$
\mathbb{E}\left(X_{n}^{2}\right)=\frac{s \Gamma(n+2 p)}{\Gamma(n) \Gamma(1+2 p)}+\frac{s p \Gamma(n+2 p)}{\Gamma(n) \Gamma(1+p)} \sum_{k=1}^{n-1} \frac{\Gamma(k+p)}{\Gamma(k+1+2 p)} .
$$


Using Lemma 2 we obtain the exact and the asymptotic formulas

$$
\begin{aligned}
\mathbb{E}\left(X_{n}^{2}\right) & =\frac{2 s \Gamma(n+2 p)}{\Gamma(n) \Gamma(1+2 p)}-\frac{s \Gamma(n+p)}{\Gamma(n) \Gamma(1+p)} . \\
& \sim \frac{2 s n^{2 p}}{\Gamma(1+2 p)}
\end{aligned}
$$

Let us apply the same ideas to compute $\mathbb{E}\left(X_{n}^{3}\right)$. It follows from 19 that

$$
\mathbb{E}\left(X_{n+1}^{3} \mid \mathcal{F}_{n}\right)=X_{n}^{3}\left(\frac{n+3 p}{n}\right)+3 p \frac{X_{n}^{2}}{n}+p \frac{X_{n}}{n},
$$

which yields

$$
\mathbb{E}\left(X_{n+1}^{3}\right)=\mathbb{E}\left(X_{n}^{3}\right)\left(\frac{n+3 p}{n}\right)+3 p \frac{\mathbb{E}\left(X_{n}^{2}\right)}{n}+p \frac{\mathbb{E}\left(X_{n}\right)}{n}
$$

Induction gives us the following expression

$$
\begin{aligned}
\mathbb{E}\left(X_{n}^{3}\right)= & \frac{s \Gamma(n+3 p)}{\Gamma(n) \Gamma(1+3 p)}+\frac{6 s p \Gamma(n+3 p)}{\Gamma(n) \Gamma(1+2 p)} \sum_{k=1}^{n-1} \frac{\Gamma(k+2 p)}{\Gamma(k+1+3 p)} \\
& -\frac{2 s p \Gamma(n+3 p)}{\Gamma(n) \Gamma(1+p)} \sum_{k=1}^{n-1} \frac{\Gamma(k+p)}{\Gamma(k+1+3 p)} .
\end{aligned}
$$

Apply Lemma 2 to the middle and right terms above to get

$$
\begin{aligned}
\mathbb{E}\left(X_{n}^{3}\right) & =\frac{s \Gamma(n+3 p)}{\Gamma(n) \Gamma(1+3 p)}+\left(\frac{6 s \Gamma(n+3 p)}{\Gamma(n) \Gamma(1+3 p)}-\frac{6 s \Gamma(n+2 p)}{\Gamma(n) \Gamma(1+2 p)}\right) \\
& -\left(\frac{s \Gamma(n+3 p)}{\Gamma(n) \Gamma(1+3 p)}-\frac{s \Gamma(n+p)}{\Gamma(n) \Gamma(1+p)}\right) \\
& =\frac{6 s \Gamma(n+3 p)}{\Gamma(n) \Gamma(1+3 p)}-\frac{6 s \Gamma(n+2 p)}{\Gamma(n) \Gamma(1+2 p)}+\frac{s \Gamma(n+p)}{\Gamma(n) \Gamma(1+p)} \\
& \sim \frac{6 s n^{3 p}}{\Gamma(1+3 p)} .
\end{aligned}
$$


Repeating all over again to $\mathbb{E}\left(X_{n}^{4}\right)$, we obtain

$$
\begin{aligned}
\mathbb{E}\left(X_{n}^{4}\right) & =\frac{s \Gamma(n+4 p)}{\Gamma(n) \Gamma(1+4 p)}+\frac{36 s p \Gamma(n+4 p)}{\Gamma(n) \Gamma(1+3 p)} \sum_{k=1}^{n-1} \frac{\Gamma(k+3 p)}{\Gamma(k+1+4 p)} \\
& -\frac{28 s p \Gamma(n+4 p)}{\Gamma(n) \Gamma(1+2 p)} \sum_{k=1}^{n-1} \frac{\Gamma(k+2 p)}{\Gamma(k+1+4 p)}+\frac{3 s p \Gamma(n+4 p)}{\Gamma(n) \Gamma(1+p)} \sum_{k=1}^{n-1} \frac{\Gamma(k+p)}{\Gamma(k+1+4 p)} \\
& =\frac{s \Gamma(n+4 p)}{\Gamma(n) \Gamma(1+4 p)}+\left(\frac{36 s \Gamma(n+4 p)}{\Gamma(n) \Gamma(1+4 p)}-\frac{36 s \Gamma(n+3 p)}{\Gamma(n) \Gamma(1+3 p)}\right) \\
& -\left(\frac{14 s \Gamma(n+4 p)}{\Gamma(n) \Gamma(1+4 p)}-\frac{14 s \Gamma(n+2 p)}{\Gamma(n) \Gamma(1+2 p)}\right)+\left(\frac{s \Gamma(n+4 p)}{\Gamma(n) \Gamma(1+4 p)}-\frac{s \Gamma(n+p)}{\Gamma(n) \Gamma(1+p)}\right) \\
& =\frac{24 s \Gamma(n+4 p)}{\Gamma(n) \Gamma(1+4 p)}-\frac{36 s \Gamma(n+3 p)}{\Gamma(n) \Gamma(1+3 p)}+\frac{14 s \Gamma(n+2 p)}{\Gamma(n) \Gamma(1+2 p)}-\frac{s \Gamma(n+p)}{\Gamma(n) \Gamma(1+p)} \\
& \sim \frac{24 s n^{4 p}}{\Gamma(1+4 p)} .
\end{aligned}
$$

\subsubsection{Almost sure convergence.}

Proof of Theorem 4. We begin with our martingale difference. Recall that $\left|D_{j}\right| \leq \frac{2}{a_{j}}$ (see (8)). Then apply the Burkholder's inequality (see Theorem 8 above) for $m>1$ to get

$$
\mathbb{E}\left(\left|M_{n}\right|^{m}\right) \leq c \mathbb{E}\left(\left|\sum_{j=1}^{n} D_{j}^{2}\right|^{m / 2}\right),
$$

where $c$ is a positive constant depending on $m$ only. Along with Lemma 1 for $p>1 / 2$ the previous inequality implies that $\sup \mathbb{E}\left(\left|M_{n}\right|^{m}\right)<\infty$, and it follows from the $L^{m}$ convergence theorem for martingales that $M_{n} \rightarrow M$ a.s. and in $L^{m}$. Moreover,

$$
|\mathbb{E}(M)|=\left|\mathbb{E}\left(M-M_{n}\right)\right| \leq \mathbb{E}\left(\left|M-M_{n}\right|\right) \leq \mathbb{E}\left(\left|M-M_{n}\right|^{2}\right)^{1 / 2} \rightarrow 0 \text { as } n \rightarrow \infty .
$$

By (20) we note that

$$
\mathbb{E}\left(M^{2}\right)=\lim _{n \rightarrow \infty} \mathbb{E}\left(\left(\frac{X_{n}}{a_{n}}\right)^{2}\right)-s^{2}=\frac{2 s \Gamma(1+p)^{2}}{\Gamma(1+2 p)}-s^{2},
$$

where $a_{n} \sim n^{p} / \Gamma(1+p)$. In order to arrive at the conclusion of the theorem, let us compute the third and fourth moment of $M$ and verify that they do not correspond to the third and fourth moment of a normally distributed random variable. Applying (5), 20), (21) and (22), we obtain

$$
\begin{aligned}
\mathbb{E}\left(M^{3}\right) & =\lim _{n \rightarrow \infty}\left\{\mathbb{E}\left(\left(\frac{X_{n}}{a_{n}}\right)^{3}\right)-3 s \mathbb{E}\left(\left(\frac{X_{n}}{a_{n}}\right)^{2}\right)+3 s^{2} \mathbb{E}\left(\frac{X_{n}}{a_{n}}\right)-s^{3}\right\} \\
& =\frac{6 s \Gamma(1+p)^{3}}{\Gamma(1+3 p)}-\frac{6 s^{2} \Gamma(1+p)^{2}}{\Gamma(1+2 p)}+2 s^{3}
\end{aligned}
$$


and

$$
\begin{aligned}
\mathbb{E}\left(M^{4}\right) & =\lim _{n \rightarrow \infty}\left\{\mathbb{E}\left(\left(\frac{X_{n}}{a_{n}}\right)^{4}\right)-4 s \mathbb{E}\left(\left(\frac{X_{n}}{a_{n}}\right)^{3}\right)+6 s^{2} \mathbb{E}\left(\left(\frac{X_{n}}{a_{n}}\right)^{2}\right)-4 s^{3} \mathbb{E}\left(\frac{X_{n}}{a_{n}}\right)+s^{4}\right\} \\
& =\frac{24 s \Gamma(1+p)^{4}}{\Gamma(1+4 p)}-\frac{24 s^{2} \Gamma(1+p)^{3}}{\Gamma(1+3 p)}+\frac{12 s^{3} \Gamma(1+p)^{2}}{\Gamma(1+2 p)}-3 s^{4},
\end{aligned}
$$

which completes the proof of Theorem 4.

\section{REFERENCES}

[1] Arita, C.; Ragoucy, E. Interacting elephant random walks. Phys. Rev. E 98, 052118 (2018).

[2] Baur, E.; Bertoin, J. Elephant random walks and their connection to Pólia-type urns. Phys. Rev. E 94, 052134 (2016).

[3] Bercu, B. A martingale approach for the elephant random walk. J. Phys. A: Math. Theor. 51(1), 015201 (2018)

[4] Budini, A.A. Memory-induced diffusive-superdiffusive transition: Ensemble and time-averaged observables Phys. Rev. E 95, 052110 (2017).

[5] Coletti, BC.F.; Gava, R.J.; Schütz, G. M. Central limit theorem for the elephant random walk. J. Math. Phys 58, 053303 (2017).

[6] Coletti, BC.F.; Gava, R.J.; Schütz, G. M. A strong invariance principle for the elephant random walk. J. Stat. Mech. Theory Exp., 123207 (2017).

[7] da Silva, M.A.A., Cressoni, J.C., Schütz, G.M., Viswanathan, G.M., Trimper, S., Non-Gaussian propagator for elephant random walks. Phys. Rev. E 88, 022115 (2013)

[8] Hall, P.; Heyde, C.C. Martingale Limit Theory and Its Application. Academic Press, New York (1980).

[9] Kearney, M.J., Martin, R.J. Random walks exhibiting anomalous diffusion: elephants, urns and the limits of normality. J. Stat. Mech., 013209 (2018).

[10] Khoromskaia, D.; Harris, R.J; Grosskinsky, S. Dynamics of non-Markovian exclusion processes J. Stat. Mech., P12013 (2014).

[11] Kumar, N., Harbola, U., Lindenberg, K., Memory-induced anomalous dynamics: Emergence of diffusion, subdiffusion, and superdiffusion from a single random walk model. Phys. Rev. E 82, 021101 (2010).

[12] Kumar, N., Harbola, U., Lindenberg, K., Memory-induced anomalous dynamics in a minimal random walk model. Phys. Rev. E 90, 022136 (2014).

[13] Kumar, N., Harbola, U., Memory induced anomalous dynamics in a random walker with internal states. J. Stat. Mech., 103207 (2018)

[14] Kürsten, R. Random recursive trees and the elephant random walk. Phys. Rev. E 93, 032111 (2016).

[15] Schütz, G. M.; Trimper, S. Elephants can always remember: Exact long-range memory effects in a nonMarkovian random walk. Phys. Rev. E 70, 045101 (2004).

[16] Stout, W. F. A martingale analogue of Kolmogorov's law of the Iterated logarithm. Z. Wahrscheinlichkeitstheorie 15, 279-290 (1970).

[17] Stout, W. F. Almost sure convergence, Probability and Mathematical Statistics, Academic Press, New York-London (1974).

[18] Williams, D. Probability with Martingales. Cambridge University Press, Cambridge (1991). 
UfabC - Centro de Matemática, Computação e Cognição.

Avenida dos Estados, 5001, Santo André - São Paulo, Brasil

E-MAIL: cristian.coletti@ufabc.edu.br

e-mail: lucas.roberto@ufabc.edu.br

UFSCAR - Departamento de Estatística.

Rodovia Washington Luiz, Km 235, CEP 13565-905, São Carlos, Brasil

e-mail: gava@ufscar.br 